\title{
Videira 'Syrah' sobre diferentes porta-enxertos em ciclo de inverno no sul de Minas Gerais
}

\author{
Frederico Alcântara Novelli Dias ${ }^{(1)}$, Renata Vieira da Mota(2), Ana Carolina Fávero(3), Eduardo Purgatto(4), \\ Tânia Misuzu Shiga ${ }^{(4)}$, Claudia Rita de Souza(2), Rodrigo Meirelles de Azevedo Pimentel(2) \\ e Murillo de Albuquerque Regina(2)
}

\begin{abstract}
(1) Universidade Federal de Lavras, Departamento de Agricultura, Caixa Postal 3037, CEP $37200-000$ Lavras, MG. E-mail: frederico_novelli190@hotmail.com (2)Empresa de Pesquisa Agropecuária de Minas Gerais, Núcleo Tecnológico Uva e Vinho, Avenida Santa Cruz, o 500, Caixa Postal 33, CEP 37780-000 Caldas, MG. E-mail: renata@epamigcaldas.gov.br, crsouza@epamig.br, rodrigomeirelles@epamig.br, murillo@epamigcaldas.gov.br ${ }^{(3)}$ Serviço Brasileiro de Apoio às Micro e Pequenas Empresas, Avenida Maria Antônia C. de Oliveira, № 2.903, CEP 14802-330 Araraquara, SP. E-mail: acfavero@yahoo.com.br (4)Universidade de São Paulo, Faculdade de Ciências Farmacêuticas, Departamento de Alimentos e Nutrição Experimental, Caixa Postal 66.083, CEP 05315-970 São Paulo, SP. E-mail: epurgatt@usp.br, tatymish@usp.br
\end{abstract}

Resumo - O objetivo deste trabalho foi avaliar a influência de porta-enxertos sobre as características agronômicas, ecofisiológicas e qualitativas da videira 'Syrah' manejada por meio da técnica da dupla poda. As videiras foram enxertadas nos porta-enxertos 'SO4', '110 Richter' e '1103 Paulsen', e sustentadas em sistema espaldeira sem irrigação. Foram avaliadas as características ecofisiológicas, de produção e de composição físico-química das bagas maduras em três safras (2007, 2008 e 2010). Os porta-enxertos não exerceram influência sobre o potencial hídrico de base, que apresentou valores próximos a $-0,2 \mathrm{MPa}$, o que indica que não houve restrição hídrica no solo ao final da maturação (junho). Também não houve diferença significativa quanto à produção. O porta-enxerto '1103 Paulsen' conferiu menor vigor, menor taxa fotossintética e melhores resultados de maturação, para as safras com menores precipitações. Os porta-enxertos '110 Richter' e 'SO4' apresentaram maior vigor nas condições meteorológicas de 2010 e as maiores taxas fotossintéticas para o mesmo período. As condições meteorológicas tiveram efeito significativo na maturação tecnológica e fenólica, com os melhores resultados para os anos mais secos. O porta-enxerto '1103 Paulsen' fornece melhor equilíbrio entre vigor e produção, o que aumenta a qualidade da uva.

Termos para indexação: Vitis vinifera, ácidos orgânicos, açúcares redutores, compostos fenólicos, manejo, produção.

\section{'Syrah' vine on different rootstocks in winter cycle in the south of Minas Gerais State, Brazil}

\begin{abstract}
The objective of this work was to evaluate rootstock influence on agronomical, ecophysiological and qualitative characteristics of 'Syrah' vines managed by double pruning. Grapevines were grafted onto 'SO4', '110 Richter' and '1103 Paulsen' rootstocks and trained in vertical shoot position, with no irrigation. Ecophysiological characteristics, yield and composition of ripe grapes were evaluated in three crop seasons (2007, 2008 and 2010). Rootstocks did not affect pre-dawn water potential, with values close to $-0.2 \mathrm{MPa}$, indicating that there was no soil water deficit at the end of ripening (June). There was also no significant effect of rootstocks on yield. The rootstock '1103 Paulsen' induced lower vegetative growth, lower photosynthetic rate and the best results for berry maturation for crop seasons with lower amount of rainfall. The rootstocks '110 Richter' and 'SO4' showed higher vigor under the meteorological conditions of 2010 and the greatest photosynthetic rates in the same period. Meteorological conditions significantly affected technological and phenolic ripeness, with best results observed in drought years. The '1103 Paulsen' rootstock provides better balance between vigor and yield, increasing grape quality.
\end{abstract}

Index terms: Vitis vinifera, organic acids, reducing sugars, phenolic compounds, management, yield.

\section{Introdução}

O cultivo de uvas viníferas para elaboração de vinhos finos em Minas Gerais é uma prática recente. Os primeiros resultados positivos de produção de uvas com maturação tecnológica adequada à vinificação foram obtidos por Amorim et al. (2005) e Favero et al. (2008) com a cultivar Syrah, manejada com a técnica da dupla poda, na região cafeeira do sul do estado. Essa prática visa transferir a época de maturação das bagas

Pesq. agropec. bras., Brasília, v.47, n.2, p.208-215, fev. 2012 
para o período de inverno, que coincide com o menor índice pluvial no Sudeste brasileiro (Regina et al., 2006).

Embora os trabalhos de Amorim et al. (2005) e Favero et al. (2008) tenham mostrado a viabilidade do cultivo da videira 'Syrah' na região sul de Minas Gerais, com produtividade de 7,0 $\mathrm{Mg} \mathrm{ha}^{-1}$, em vinhedo não irrigado, variáveis relativas ao desempenho agronômico, como porta-enxertos, densidade, clones e sistema de condução, precisam ser testadas antes de se preconizar um pacote tecnológico para a cultura.

Por se tratar de nova região produtora de uvas finas, não há dados na literatura sobre a influência de porta-enxertos na produção e na qualidade de bagas da uva 'Syrah' cultivada em Minas Gerais.

De modo geral, a indicação de porta-enxertos baseia-se na melhor adaptação às condições ambientais e na compatibilidade com a copa, o que afeta diretamente a produtividade e algumas características químicas da baga, como $\mathrm{pH}$, acidez e teor de sólidos solúveis ( ${ }^{\circ}$ Brix). Além disso, a absorção de nutrientes, o acúmulo de compostos fenólicos e o teor de antocianinas são parâmetros de qualidade que também devem ser levados em conta na escolha da melhor combinação copa/ porta-enxerto (Jackson \& Lombard, 1993).

O objetivo deste trabalho foi avaliar a influência de porta-enxertos sobre as caraterísticas agronômicas, ecofisiológicas e qualitativas da videira 'Syrah' manejada por meio da técnica da dupla poda.

\section{Material e Métodos}

O experimento foi realizado em vinhedo implantado em outubro de 2003, na Fazenda da Fé, situada no

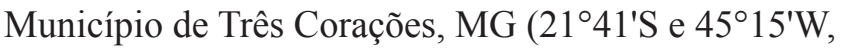
a $900 \mathrm{~m}$ de altitude). O clima da região é do tipo $\mathrm{Cwa}$, temperado quente, conforme a classificação de Köppen, com temperatura máxima anual de $26,7^{\circ} \mathrm{C}$, em média, e amplitude térmica diária de $15^{\circ} \mathrm{C}$ de junho a agosto. A época mais seca, com duração de três a quatro meses, coincide com o inverno, e há ocorrência de pelo menos um mês com precipitação, em média, inferior a $60 \mathrm{~mm}$ (Tonietto et al., 2006).

$\mathrm{O}$ experimento foi instalado em área de Latossolo Vermelho-Amarelo (Santos et al., 2006) contendo 300 plantas, em espaçamento de 2,5x1,5 m, com mudas da cultivar Syrah, clone 747, provenientes do Etablissement National Technique pour l'Amélioration de la Viticulture - Institut National de la Recherche
Agronomique (ENTAV INRA). Utilizou-se o delineamento inteiramente casualizado com três tratamentos, constituídos pelos porta-enxertos '1103 Paulsen' (Vitis berlandieri x V. rupestris), 'SO4' (V. berlandieri x V. riparia) e '110 Richter' ( $V$. berlandieri $\mathrm{x} V$. rupestris). Cada tratamento foi composto por 100 plantas.

Nos três tratamentos, as plantas foram conduzidas em duplo cordão esporonado, no suporte espaldeira, sem irrigação. Efetuou-se poda de formação de ramos, em setembro, e poda de produção, em janeiro, conforme metodologia preconizada para dupla poda por Regina et al. (2006).

As avaliações quantitativas e qualitativas das bagas foram realizadas nas safras de 2007, 2008 e 2010. No entanto, as avaliações das características ecofisiológicas, agronômicas e de produção foram realizadas apenas em 2010, quando a arquitetura das plantas estava completamente formada. Para a safra de 2009 , não foram realizadas avaliações, em virtude da forte chuva de granizo que atingiu o vinhedo durante o período de formação dos ramos, o que comprometeu a produção. A precipitação observada durante o período de maturação das bagas nas safras de 2007, 2008 e 2010 foi de 111, 43 e $51 \mathrm{~mm}$, respectivamente.

Os potenciais hídricos do caule $\left(\Psi_{\text {caule }}\right)$ e de base ( $\left.\Psi_{\text {base }}\right)$ foram avaliados com câmara de Scholander, modelo 3005 (Soilmoisture Equipment Corp., Santa Barbara, CA, EUA), tendo-se utilizado nitrogênio como gás inerte. $\mathrm{O} \Psi_{\text {base }}$ foi medido antes do amanhecer, enquanto o $\Psi_{\text {caule }}$ foi avaliado no final da manhã (11h) em seis folhas embaladas por, pelo menos, 1 hora em filme plástico e papel alumínio (Choné et al., 2001).

As avaliações da taxa fotossintética e da abertura estomática foram realizadas com analisador de gás por infravermelho, IRGA, (LI-6400, Licor, Lincoln, NE, EUA), em condições de luminosidade acima do ponto de saturação $\left(1.000 \mu \mathrm{mol} \mathrm{m} \mathrm{m}^{-2} \mathrm{~s}^{-1}\right)$, às $10 \mathrm{~h}$ da manhã, em seis folhas sadias, maduras, da porção mediana dos ramos e totalmente expostas à radiação solar.

A superfície foliar primária foi avaliada em cinco plantas durante a maturação das bagas, após o desponte das plantas, na segunda quinzena de maio, pelo método não destrutivo, conforme Regina et al. (2000).

As avaliações físicas foram realizadas na colheita. Um lote de 50 bagas de cada tratamento foi utilizado para a determinação do diâmetro transversal, com auxílio de paquímetro manual. A massa dos cachos foi determinada pela pesagem de dez cachos por 
tratamento com dez repetições, e a massa das bagas foi obtida a partir da pesagem de 50 bagas em cinco repetições. O número de bagas por cacho foi contado em amostragem de 100 cachos por tratamento. O valor da massa dos cachos foi multiplicado pelo número de cachos de cada planta, para a determinação da produção média estimada por planta para cada tratamento. Para a determinação da produção estimada por hectare, multiplicou-se a produção média por planta de cada tratamento pelo número de plantas por hectare. A relação área foliar por quilograma de fruto foi estimada pela razão entre os valores da área foliar primária e produção média estimada por planta.

A composição química do mosto foi avaliada em amostra composta por 210 bagas para cada tratamento. Além das análises de sólidos solúveis, acidez total titulável e $\mathrm{pH}$, foram realizadas análises dos teores de ácidos orgânicos e açúcares redutores.

Os ácidos orgânicos foram separados dos açúcares pela passagem do mosto por resina de troca aniônica Bio-Rex 5, (Bio-Rad Laboratories, Hercules, CA, EUA), conforme McCord et al. (1984). Os ácidos tartárico e málico foram quantificados em cromatógrafo líquido, modelo 1100 (Hewlett-Packard Company, Palo Alto, CA, EUA), equipado com coluna SupelcoGel C-610H de $30 \mathrm{~cm}$ x 7,8 mm (Supelco Sigma-Aldrich, Bellefonte, $\mathrm{PA}$, EUA), ajustada à temperatura de $15^{\circ} \mathrm{C}$, e com detector arranjo de diodos (DAD) a $245 \mathrm{~nm}$, em corrida isocrática a fluxo de $0,5 \mathrm{~mL} \mathrm{~min}{ }^{-1}$, tendo-se utilizado solução de ácido fosfórico a $0,5 \%$ como fase móvel.

Os teores de glicose, frutose e sacarose foram determinados por HPLC-PAD em cromatógrafo DX-500, (Thermo Scientific, Sunnyvale, CA, EUA), com coluna CarboPac PA1 de 4,0 cm x $250 \mathrm{~mm}$ (Thermo Scientific, Sunnyvale, CA, EUA), acoplado a detector de pulso amperométrico em corrida isotérmica a $25^{\circ} \mathrm{C}$. Como fase móvel, foi utilizado $\mathrm{NaOH} 18 \mathrm{mmol} \mathrm{L}^{-1} \mathrm{em}$ fluxo isocrático de $1,0 \mathrm{~mL} \mathrm{~min}{ }^{-1}$.

A maturação fenólica foi avaliada pela determinação do teor de antocianinas e fenólicos totais em amostra composta de 100 bagas para cada tratamento. Uma alíquota de $250 \mathrm{mg}$ de casca triturada em nitrogênio líquido foi homogeneizada em dispersador Ultra Turrax T 18 basic, (IKA, Wilmington, NC, EUA), em solução extratora constituída de metanol acidificado ( $\mathrm{HCl} 1 \%)$. As antocianinas presentes nas variedades tintas foram determinadas pelo método do $\mathrm{pH}$ diferencial (Giusti \& Wrolstad, 2000), e os fenólicos totais foram analisados pelo método de Folin-Ciocalteau com base em curva padrão de ácido gálico (Amerine \& Ough, 1980).

As sementes foram imersas por 48 horas, à temperatura ambiente e ao abrigo da luz, em solução alcoólica composta por metanol acidificado ( $\mathrm{HCl} 1 \%)$, em proporção correspondente ao volume de mosto das bagas (González-Neves et al., 2004). Os compostos fenólicos extraídos foram determinados pelo método de Folin-Ciocalteau. O teor de potássio foi obtido por fotometria de chama após digestão nítrico-perclórica das bagas (Malavolta et al., 1997). O vigor foi determinado pela massa seca dos ramos de seis plantas oriundos da poda de inverno dois meses após a colheita.

Os dados foram submetidos à análise de variância, com auxílio do programa Sisvar (Ferreira, 2008), e as médias foram comparadas pelo teste de Tukey, a $5 \%$ de probabilidade.

\section{Resultados e Discussão}

A produtividade observada para os três tratamentos na safra de 2010 aproxima-se de $12 \mathrm{Mg} \mathrm{ha}^{-1}$ (Tabela 1), valor considerado favorável à elaboração de vinhos finos de qualidade, para a cultivar Syrah, na região de altitude de São Joaquim, SC (Silva et al., 2009). Além disso, a produção de 3,3 a 3,9 kg por planta assemelha-se à obtida para o clone 100 da variedade Syrah enxertado sobre o porta-enxerto 'SO4', o mais produtivo nos estudos de Regina \& Audeguin (2005), na França.

Em geral, os tratamentos apresentaram diferença em relação ao vigor, com menor produção de biomassa nas videiras sobre o porta-enxerto '1103 Paulsen'.

A relação área foliar por quilograma de fruto observada nos três tratamentos de 0,5 a $0,8 \mathrm{~m}^{2} \mathrm{~kg}^{-1}$ está na faixa proposta por Kliewer \& Dokoozlian (2005),

Tabela 1. Parâmetros produtivos e de vigor vegetativo da videira 'Syrah' enxertada em três porta-enxertos, na safra de 2010, na região de Três Corações, $\mathrm{MG}^{(1)}$.

\begin{tabular}{lccc}
\hline Variável & \multicolumn{3}{c}{ Porta-enxerto } \\
\cline { 2 - 4 } & '1103 Paulsen' & '110 Richter' & 'SO4' \\
\hline Produção média $\left(\mathrm{kg} \mathrm{planta}^{-1}\right)$ & $3,29 \mathrm{a}$ & $3,86 \mathrm{a}$ & $3,88 \mathrm{a}$ \\
Produtividade $\left(\mathrm{Mg} \mathrm{ha}^{-1}\right)$ & $10,15 \mathrm{a}$ & $11,91 \mathrm{a}$ & $11,97 \mathrm{a}$ \\
Massa da poda $\left(\mathrm{g} \mathrm{planta}{ }^{-1}\right)$ & $223,54 \mathrm{~b}$ & $290.96 \mathrm{a}$ & $288,62 \mathrm{a}$ \\
Área foliar $\left(\mathrm{m}^{2}\right)$ & $2,60 \mathrm{a}$ & $2,73 \mathrm{a}$ & $3,34 \mathrm{a}$ \\
Relação área foliar:produção $\left(\mathrm{m}^{2} \mathrm{~kg}^{-1}\right)$ & $0,80 \mathrm{a}$ & $0,71 \mathrm{a}$ & $0,86 \mathrm{a}$ \\
\hline \multirow{3}{*}{${ }^{(1)}$ Médias seguidas de letras iguais, nas linhas, não diferem, entre si, pelo } \\
teste de Tukey, a 5\% de probabilidade.
\end{tabular}


considerada como necessária para a boa maturação dos frutos.

Para a safra de 2010, os porta-enxertos não exerceram influência sobre os parâmetros agronômicos quantitativos da videira 'Syrah'. Isso corrobora os resultados de Koundouras et al. (2008), que afirmam que a produção sofre maior influência do estado hídrico da planta do que do tipo de porta-enxerto utilizado. O monitoramento do estado hídrico do vinhedo foi realizado por meio da avaliação sazonal do $\Psi_{\text {caule }}$ e da avaliação do $\Psi_{\text {base }}$ obtido ao final da maturação dos frutos (Tabela 2). Os valores do $\Psi_{\text {caule }}$ registrados não são indicativos de deficiência hídrica no vinhedo (Koundouras et al., 2008).

Os valores do $\Psi_{\text {base }}$ observados em todos os tratamentos indicam que o vinhedo, ao final da maturação, encontrava-se com boa disponibilidade hídrica no solo (Deloire et al., 2004) e que não houve efeito dos porta-enxertos no estado hídrico das plantas da cultivar Syrah. Esses resultados confirmam a observação de Amorim et al. (2005) de que, durante o ciclo de outono/inverno, a ocorrência de maior volume das precipitações até o início da maturação, somado ao fato de os solos com vocação agrícola da região de Três Corações serem argilosos e reterem muita água, é suficiente para atender às necessidades hídricas da videira 'Syrah'.

Apesar do estado hídrico da 'Syrah' não ter sido influenciado pelos porta-enxertos na maioria dos estádios fenológicos avaliados, o '1103 Paulsen' induziu redução significativa de $21 \%$ na taxa fotossintética das videiras, conforme valores observados ao final da

Tabela 2. Potencial hídrico de base ( $\left.\Psi_{\text {base }}\right)$, taxa fotossintética (A) e abertura estomática (gs), obtidos ao final da maturação das bagas, e potencial hídrico do caule $\left(\Psi_{\text {caule }}\right)$ avaliado em três estágios fenológicos da videira 'Syrah' enxertada em três porta-enxertos, na safra de 2010, na região de Três Corações, $\mathrm{MG}^{(1)}$.

\begin{tabular}{lccc}
\hline Variável & \multicolumn{3}{c}{ Porta-enxerto } \\
\cline { 2 - 4 } & '1103 Paulsen' & '110 Richter' & 'SO4' \\
\hline$\Psi_{\text {base }}(\mathrm{Mpa})$ & $-0,26 \mathrm{a}$ & $-0,22 \mathrm{a}$ & $-0,26 \mathrm{a}$ \\
$\mathrm{A}\left(\mu \mathrm{mol} \mathrm{CO} \mathrm{m}^{-2} \mathrm{~s}^{-1}\right)$ & $10,00 \mathrm{~b}$ & $13,32 \mathrm{a}$ & $12,09 \mathrm{ab}$ \\
$\mathrm{gs}\left(\mathrm{mol} \mathrm{m}^{-2} \mathrm{~s}^{-1}\right)$ & $0,16 \mathrm{a}$ & $0,22 \mathrm{a}$ & $0,21 \mathrm{a}$ \\
$\Psi_{\text {caule }}(\mathrm{Mpa})$ & & & \\
$\quad$ Abril (cacho fechado) & $-0,52 \mathrm{a}$ & $-0,41 \mathrm{a}$ & $-0,49 \mathrm{a}$ \\
$\quad$ Maio (pintor) & $-0,37 \mathrm{a}$ & $-0,37 \mathrm{a}$ & $-0,39 \mathrm{a}$ \\
$\quad$ Junho (maturação) & $-0,66 \mathrm{a}$ & $-0,65 \mathrm{a}$ & $-0,61 \mathrm{a}$ \\
\hline
\end{tabular}

${ }^{(1)}$ Médias seguidas de letras iguais, nas linhas, não diferem, entre si, pelo teste de Tukey, a 5\% de probabilidade. maturação, quando comparado aos demais tratamentos (Tabela 2). Essa redução pode ser atribuída à maior restrição à difusão do $\mathrm{CO}_{2}$ para o interior dos cloroplastos, em virtude do maior fechamento estomático (menor gs) ocorrido nas videiras enxertadas sobre o '1103 Paulsen'. Contudo, não foram observadas diferenças significativas entre os tratamentos.

Plantas enxertadas sobre'1103 Paulsen' apresentaram menor número de bagas por cacho. Entretanto, não houve diferença significativa na massa dos cachos entre os tratamentos, em razão da maior massa das bagas nas videiras sobre '1103 Paulsen'.

Amorim et al. (2005) observaram massa média dos cachos da cultivar Syrah enxertada sobre o porta-enxerto '3309 C' de 160 g, enquanto Favero et al. (2008) obtiveram, na mesma área experimental, massas de 111,15 e 142,56 g nas safras de 2005 e 2006, respectivamente. A safra de 2010 apresentou massa média dos cachos acima dos valores encontrados por esses autores, o que mostra o maior potencial produtivo do vinhedo com sete anos de idade (Tabela 3 ).

Os valores de massa das bagas inferior a 2,0 g estão na faixa considerada como de bagas pequenas (Rizzon $\&$ Miele, 2004). O tamanho das bagas é um dos fatores que determina a qualidade da uva vinífera, notadamente para elaboração de vinhos tintos, uma vez que, nas bagas menores, há maior relação soluto:solvente e, consequentemente, maior probabilidade de extração

Tabela 3. Parâmetros físicos dos cachos e das bagas da videira 'Syrah' enxertada em três porta-enxertos, para as safras de 2007, 2008 e 2010, na região de Três Corações, MG $^{(1)}$.

\begin{tabular}{lccc}
\hline Safra & \multicolumn{3}{c}{ Porta-enxerto } \\
\cline { 2 - 4 } & '1103 Paulsen' & '110 Richter' & 'SO4' \\
\hline \multicolumn{4}{c}{ Massa do cacho $(\mathrm{g})$} \\
2008 & $153,54 \mathrm{aB}$ & $161,58 \mathrm{aB}$ & $164,00 \mathrm{aB}$ \\
2010 & $201,82 \mathrm{aA}$ & $212,02 \mathrm{aA}$ & $217,23 \mathrm{aA}$ \\
\hline \multicolumn{4}{c}{ No bagas por cacho } \\
2008 & $156 \mathrm{bA}$ & $185 \mathrm{aA}$ & $185 \mathrm{aA}$ \\
2010 & $129 \mathrm{bB}$ & $160 \mathrm{aB}$ & $165 \mathrm{aB}$ \\
\hline \multicolumn{4}{c}{ Massa da baga (g) } \\
2007 & $1,55 \mathrm{aAB}$ & $1,38 \mathrm{aB}$ & $1,44 \mathrm{aA}$ \\
2008 & $1,41 \mathrm{aB}$ & $1,30 \mathrm{bB}$ & $1,20 \mathrm{cB}$ \\
2010 & $1,62 \mathrm{aA}$ & $1,59 \mathrm{aA}$ & $1,57 \mathrm{aA}$ \\
\hline \multicolumn{4}{c}{ Diâmetro transversal (mm) } \\
2007 & $13,88 \mathrm{aA}$ & $13,04 \mathrm{bA}$ & $12,72 \mathrm{bB}$ \\
2008 & $13,70 \mathrm{aA}$ & $12,71 \mathrm{bA}$ & $13,35 \mathrm{aA}$ \\
2010 & $11,30 \mathrm{abB}$ & $11,73 \mathrm{aB}$ & $11,15 \mathrm{bC}$ \\
\hline
\end{tabular}

(1)Médias seguidas de letras iguais, minúsculas nas linhas e maiúsculas nas colunas, não diferem, entre si, pelo teste de Tukey, a 5\% de probabilidade. 
de minerais, antocianinas e outros compostos fenólicos das cascas durante a maceração (Conde et al., 2007).

A safra de 2010 apresentou os menores tamanhos das bagas, provavelmente em função da influência das condições climáticas no seu desenvolvimento. $\mathrm{Na}$ média dos tratamentos para as três safras, os valores encontraram-se muito próximos: $12,90 \mathrm{~mm}$, para '1103 Paulsen', 12,49 mm, para '110 Richter', e 12,41 mm para 'SO4', o que indica que o tamanho das bagas sofre maior influência das características climáticas de cada safra do que dos porta-enxertos.

$\mathrm{O}$ teor de sólidos solúveis foi, na média dos tratamentos, de $20^{\circ}$ Brix em 2007, $21^{\circ}$ Brix em 2008 e $20^{\circ}$ Brix em 2010 (Tabela 4). Esses teores são inferiores, porém próximos, à faixa observada para essa cultivar na Austrália (Ginestar et al., 1998) e na Espanha (Ortega-Regules et al., 2008), locais com elevadas temperaturas médias e, portanto, maior taxa fotossintética, o que pode ter ocasionado o maior acúmulo de açúcares relatado por esses autores.

O decreto 99066/1990, da legislação brasileira, determina que o teor alcoólico dos vinhos deve estar na faixa de 8,6 a $14^{\circ} \mathrm{GL}$ (Brasil, 1990). Com base no cálculo citado por Benavent \& Sánchez (1999) para conversão da quantidade de açúcar do mosto em grau alcoólico provável, o mosto obtido das uvas nos três tratamentos apresentou, nas três safras, grau alcoólico provável na faixa de 10,3 a $11,7^{\circ} \mathrm{GL}$, o que indica que a vinificação pode ser realizada sem a necessidade de chaptalização para as uvas viníferas cultivadas sobre os três porta-enxertos.

Nas três safras avaliadas, o teor de glicose no mosto foi ligeiramente superior ao de frutose, o que mostra que as uvas não atingiram o estágio de plena maturação das bagas (Blouin \& Guimberteau, 2002). O pH do mosto foi maior na safra de 2008, especialmente nas uvas enxertadas sobre '1103 Paulsen' e 'SO4'. A análise dos teores de potássio nas bagas indicou maior absorção deste elemento nas plantas enxertadas em '1103 Paulsen', na safra de 2008, o que pode ter contribuído para o aumento observado no $\mathrm{pH}$. Main et al. (2002) também observaram influência dos porta-enxertos nos teores de $\mathrm{pH}$ e potássio nas bagas e no vinho da cultivar Chardonel, em Fayetteville, AR, EUA. Plantas enxertadas em '110 Richter' apresentaram bagas com $\mathrm{pH}$ inferior e vinho com menor teor de $\mathrm{pH}$ e de potássio.

O teor de acidez total médio de $8,0 \mathrm{~g} \mathrm{~L}^{-1}$ observado nos três tratamentos aproxima-se dos valores obtidos nas tradicionais regiões produtoras (Tabela 4). Blouin \& Guimberteau (2002) verificaram acidez total de 7,8 $\mathrm{g} \mathrm{L}^{-1}$ para a cultivar Merlot, na região de Bordeaux, na França. No Brasil, Rizzon \& Miele (2002) observaram valores médios de $9,0 \mathrm{~g} \mathrm{~L}^{-1}$, em seis safras, para a cultivar Cabernet Sauvignon, na Serra Gaúcha, RS.

As bagas sobre 'SO4' apresentaram as menores concentrações de ácido málico e tartárico para a safra

Tabela 4. Teores de sólidos solúveis, açúcares redutores, $\mathrm{pH}$, potássio, acidez titulável e ácidos orgânicos do mosto das bagas da videira 'Syrah' enxertada em três porta-enxertos, para as safras de 2007, 2008 e 2010, na região de Três Corações, $\mathrm{MG}^{(1)}$.

\begin{tabular}{|c|c|c|c|}
\hline \multirow[t]{2}{*}{ Safra } & \multicolumn{3}{|c|}{ Porta-enxerto } \\
\hline & '1103 Paulsen' & '110 Richter' & 'SO4' \\
\hline & \multicolumn{3}{|c|}{ Sólidos solúveis $\left({ }^{\circ} \mathrm{Brix}\right)$} \\
\hline 2007 & $19,70 \mathrm{bB}$ & $21,30 \mathrm{aA}$ & $19,63 b B$ \\
\hline 2008 & $22,13 \mathrm{aA}$ & $21,00 \mathrm{bA}$ & $21,07 \mathrm{bA}$ \\
\hline \multirow[t]{2}{*}{2010} & $20,04 \mathrm{aB}$ & $20,03 \mathrm{abB}$ & $19,73 \mathrm{bB}$ \\
\hline & \multicolumn{3}{|c|}{ Glicose $\left(\mathrm{g} \mathrm{L}^{-1}\right)$} \\
\hline 2007 & $94,33 \mathrm{bB}$ & $112,22 \mathrm{aA}$ & $97,04 \mathrm{bB}$ \\
\hline 2008 & $111,88 \mathrm{aA}$ & $109,61 \mathrm{aA}$ & $113,37 \mathrm{aA}$ \\
\hline \multirow[t]{2}{*}{2010} & $105,67 \mathrm{aAB}$ & $93,96 \mathrm{aB}$ & $93,90 \mathrm{aB}$ \\
\hline & \multicolumn{3}{|c|}{ Frutose $\left(\mathrm{g} \mathrm{L}^{-1}\right)$} \\
\hline 2007 & $90,05 \mathrm{bA}$ & $101,97 \mathrm{aA}$ & $89,81 \mathrm{bAB}$ \\
\hline 2008 & $92,75 \mathrm{aA}$ & $97,05 \mathrm{aA}$ & $99,82 \mathrm{aA}$ \\
\hline \multirow[t]{2}{*}{2010} & $96,16 \mathrm{aA}$ & $90,27 \mathrm{abA}$ & $84,31 \mathrm{bB}$ \\
\hline & \multicolumn{3}{|c|}{ Glicose:frutose } \\
\hline 2007 & $1,05 \mathrm{aB}$ & $1,10 \mathrm{aA}$ & $1,08 \mathrm{aA}$ \\
\hline 2008 & $1,21 \mathrm{aA}$ & $1,13 \mathrm{bA}$ & $1,14 \mathrm{bA}$ \\
\hline \multirow[t]{2}{*}{2010} & $1,10 \mathrm{abB}$ & $1,04 \mathrm{bB}$ & $1,11 \mathrm{aA}$ \\
\hline & \multicolumn{3}{|c|}{$\mathrm{pH}$} \\
\hline 2007 & $3,59 \mathrm{aB}$ & $3,50 \mathrm{bA}$ & $3,48 \mathrm{bB}$ \\
\hline 2008 & $3,68 \mathrm{aA}$ & $3,50 \mathrm{bA}$ & $3,53 \mathrm{bA}$ \\
\hline \multirow[t]{2}{*}{2010} & $3,38 \mathrm{aC}$ & $3,28 \mathrm{bB}$ & $3,24 \mathrm{cC}$ \\
\hline & \multicolumn{3}{|c|}{ Potássio $\left(\mathrm{g} \mathrm{kg}^{-1}\right.$ de baga $)$} \\
\hline 2007 & $2,98 \mathrm{aA}$ & $2,80 \mathrm{aA}$ & $2,71 \mathrm{aA}$ \\
\hline \multirow[t]{2}{*}{$\underline{2008}$} & $2,94 \mathrm{aA}$ & $2,43 \mathrm{bB}$ & $2,48 \mathrm{bA}$ \\
\hline & \multicolumn{3}{|c|}{ Acidez titulável $\left(\mathrm{g} \mathrm{L}^{-1}\right)$} \\
\hline 2007 & $8,30 \mathrm{bA}$ & $8,72 \mathrm{aA}$ & $8,57 \mathrm{abA}$ \\
\hline 2008 & $7,05 \mathrm{bB}$ & $7,25 \mathrm{abB}$ & $7,43 \mathrm{aB}$ \\
\hline \multirow[t]{2}{*}{2010} & $7,37 \mathrm{cB}$ & $8,40 \mathrm{bA}$ & $8,90 \mathrm{aA}$ \\
\hline & \multicolumn{3}{|c|}{ Ácido tartárico $\left(\mathrm{g} \mathrm{L}^{-1}\right)$} \\
\hline 2007 & $6,18 \mathrm{aA}$ & $6,33 \mathrm{aA}$ & $6,02 \mathrm{aA}$ \\
\hline 2008 & $6,36 \mathrm{aA}$ & $6,14 \mathrm{aA}$ & $5,63 \mathrm{bAB}$ \\
\hline \multirow[t]{2}{*}{2010} & $5,29 \mathrm{aB}$ & $5,33 \mathrm{aB}$ & $5,08 \mathrm{aB}$ \\
\hline & \multicolumn{3}{|c|}{ Ácido málico $\left(\mathrm{g} \mathrm{L}^{-1}\right)$} \\
\hline 2007 & $3,52 \mathrm{aA}$ & $3,32 \mathrm{aA}$ & $3,05 \mathrm{aA}$ \\
\hline 2008 & $3,61 \mathrm{aA}$ & $2,61 \mathrm{bB}$ & $1,87 \mathrm{cB}$ \\
\hline 2010 & $3,48 \mathrm{aA}$ & $2,98 \mathrm{bAB}$ & $3,43 \mathrm{abA}$ \\
\hline
\end{tabular}

(1)Médias seguidas de letras iguais, minúsculas nas linhas e maiúsculas nas colunas, não diferem, entre si, pelo teste de Tukey, a $5 \%$ de probabilidade. 
de 2008, provavelmente por apresentarem tamanho semelhante às de '1103 Paulsen' e menor massa entre os tratamentos. Para as safras de 2007 e 2010, não foram observadas diferenças significativas nas concentrações dos ácidos orgânicos, o que confirma que esse porta-enxerto é mais sensível às restrições hídricas do solo (Cordeau, 1998).

Além da maturação tecnológica definida pelos teores de sólidos solúveis e acidez, busca-se, na produção de uvas voltadas à elaboração de vinhos finos, a maturação fenólica das bagas. O objetivo é colher a uva no ponto máximo de qualidade quanto a açúcares, ácidos e polifenóis (taninos e antocianinas), para a produção de vinhos tintos encorpados, de alta longevidade e qualidade (Conde et al., 2007).

Não houve diferença significativa nos teores de antocianinas ou fenólicos totais entre os tratamentos, com exceção do teor de antocianinas na safra de 2008, que foi significativamente menor no porta-enxerto 'SO4' (Tabela 5). Embora não tenham sido observadas diferenças significativas entre os tratamentos, '1103 Paulsen' apresentou maiores teores de antocianinas e fenólicos totais nas três safras avaliadas. $\mathrm{O}$ menor vigor conferido pelo porta-enxerto '1103 Paulsen' contribuiu para o maior acúmulo de compostos fenólicos, por ter induzido redução na área enfolhada dessas plantas, o que facilitou a entrada da luz solar na região dos cachos (Bergqvist et al., 2001). Esses resultados estão de acordo com os de

Tabela 5. Concentração de antocianinas e de compostos fenólicos nas bagas e nas sementes da videira 'Syrah' enxertada em três porta enxertos, para as safras de 2007, 2008 e 2010, em Três Corações, $\mathrm{MG}^{(1)}$.

\begin{tabular}{lccc}
\hline Safra & \multicolumn{3}{c}{ Porta-enxerto } \\
\cline { 2 - 4 } & '1103 Paulsen' & '110 Richter' & 'SO4' \\
\hline \multicolumn{4}{c}{ Antocianinas (mg por 100 g de malvidina) } \\
2007 & $165.85 \mathrm{aAB}$ & $139.97 \mathrm{aA}$ & $150,57 \mathrm{aA}$ \\
2008 & $211,46 \mathrm{aA}$ & $174.91 \mathrm{abA}$ & $143.31 \mathrm{bA}$ \\
2010 & $139,63 \mathrm{aB}$ & $114,50 \mathrm{aA}$ & $111,60 \mathrm{aA}$ \\
\hline \multicolumn{4}{c}{ Fenólicos totais nas cascas (mg por 100 g de ácido gálico) } \\
2007 & $499,99 \mathrm{aAB}$ & $359,34 \mathrm{aA}$ & $401,31 \mathrm{aA}$ \\
2008 & $591,34 \mathrm{aA}$ & $431,93 \mathrm{aA}$ & $432,43 \mathrm{aA}$ \\
2010 & $368,39 \mathrm{aB}$ & $350,80 \mathrm{aA}$ & $250,51 \mathrm{aA}$ \\
\hline \multicolumn{4}{c}{ Fenólicos totais (mg por g de ácido gálico) } \\
2007 & $75,58 \mathrm{aA}$ & $66,21 \mathrm{bA}$ & $81,77 \mathrm{aA}$ \\
2008 & $45,55 \mathrm{aC}$ & $48,31 \mathrm{aB}$ & $54,51 \mathrm{aC}$ \\
2010 & $62,20 \mathrm{aB}$ & $64,52 \mathrm{aA}$ & $66,81 \mathrm{aB}$ \\
\hline
\end{tabular}

${ }^{(1)}$ Médias seguidas de letras iguais, minúsculas nas linhas e maiúsculas nas colunas, não diferem pelo teste Tukey, a 5\% de probabilidade.
Ezzahouani \& Williams (1995), que também não observaram efeito dos porta-enxertos '1103 Paulsen', '110 Richter' ou 'SO4' na cor da casca das uvas 'Ruby Seedless', e com os de Koundouras et al. (2009), que avaliaram a influência dos porta-enxertos '1103 Paulsen' e 'SO4', para a cultivar Cabernet Sauvignon, na região central da Grécia.

Os valores de antocianinas e de compostos fenólicos nas bagas obtidos para a videira 'Syrah', nos três porta-enxertos, para as três safras (Tabela 5), encontram-se na faixa de 30 a $750 \mathrm{mg}$ por $100 \mathrm{~g}$, e de 260 a $900 \mathrm{mg}$ por $100 \mathrm{~g}$ de bagas maduras para antocianinas e compostos fenólicos, respectivamente, conforme Mazza \& Miniati (1993), o que é indicativo de bons índices de maturação fenólica para a produção de vinhos tintos finos.

Portanto, não houve diferença na produção das plantas e no estado hídrico do vinhedo, embora o porta-enxerto '1103 Paulsen' tenha conferido menor vigor vegetativo, o que pode ter ocasionado maior direcionamento dos fotoassimilados para os cachos e favorecido a qualidade da uva para as safras mais secas. O menor vigor vegetativo das plantas também influenciou a redução da acidez total pela maior exposição dos cachos à luz solar, característica importante para a produção de vinho fino de qualidade.

\section{Conclusões}

1. O porta-enxerto '1103 Paulsen' fornece melhor equilíbrio entre vigor e produção, o que aumenta a qualidade da uva.

2. O efeito das condições meteorológicas nos diferentes anos sobre a composição físico-química das bagas é mais marcante do que o de porta-enxerto.

3. A produção sofre maior influência do estado hídrico da planta do que do tipo de porta-enxerto utilizado.

\section{Agradecimentos}

À Fundação de Amparo à Pesquisa do Estado de Minas Gerais, ao Conselho Nacional de Desenvolvimento Científico e Tecnológico e à Coordenação de Aperfeiçoamento de Pessoal de Nível Superior, pelo auxílio financeiro e pela concessão de bolsas; aos proprietários da Fazenda da Fé, por ceder a área e a mão de obra para implantação e manutenção do experimento; e ao Dr. Marcos Roberto Lopes do Nascimento, por 
ceder as instalações da Comissão Nacional de Energia Nuclear de Poços de Caldas, MG, para a realização da digestão nítrico-perclórica das bagas.

\section{Referências}

AMERINE, M.A.; OUGH, C.S. Methods for analysis of musts and wines. New York: John Wiley and Sons, 1980. 341p.

AMORIM, D.A. de; FAVERO, A.C.; REGINA, M. de A. Produção extemporânea da videira, cultivar Syrah, nas condições do sul de Minas Gerais. Revista Brasileira de Fruticultura, v.27, p.327-331, 2005.

BENAVENT, J.L.A.; SÁNCHEZ, F.M. El mosto. In: BENAVENT, J.L.A.; SÁNCHEZ, F.M. Manual de enología. Valencia: Universidad Politécnica de Valencia, 1999. p.41-57.

BERGQVIST, J.; DOKOOZLIAN, N.; EBISUDA, N. Sunlight exposure and temperature effects on berry growth and composition of Cabernet Sauvignon and Grenache in the central San Joaquin Valley of California. American Journal of Enology and Viticulture, v.52, p.1-7, 2001.

BLOUIN, J.; GUIMBERTEAU, G. Principales componentes de la uva y evolución durante la maduración. In: BLOUIN, J; GUIMBERTEAU, G (Ed.). Maduración y madurez de la uva. Madri: Mundi-Prensa, 2002. p.57-100.

BRASIL. Ministério da Agricultura, Pecuária e Abastecimento. Decreto n $^{\circ} 99.066$, de 8 de março de 1990. Dispõe sobre a produção, circulação e comercialização do vinho e derivados do vinho e de uva. Diário Oficial [da] República Federativa do Brasil, 9 mar. 1990. Seção 1, p.4755.

CHONÉ, X.; LEEUWEN, C. van; DUBOURDIEU, D.; GAUDILLÈRE, J.P. Stem water potential is a sensitive indicator of grapevine water status. Annals of Botany, v.87, p.477-483, 2001.

CONDE, C.; SILVA, P.; FONTES, N.; DIAS, A.C.P.; TAVARES, R.M.; SOUSA, M.J.; AGASSE, A.; DELROT, S.; GERÓS, H. Biochemical changes throughout grape berry development and fruit and wine quality. Food, v.1, p.1-22, 2007.

CORDEAU, J. L'influence du porte-greffe sur le comportement de la vigne. In: CORDEAU, J. (Ed.). Création d'un vignoble: greffage de la vigne et porte-greffes, élimination des maladies à virus. Bordeaux: Féret, 1998. p.83-90.

DELOIRE, A.; CARBONNEAU, A.; WANG, Z.; OJEDA, H. Vine and water: a short review. Journal International des Sciences de la Vigne et du Vin, v.38, p.1-13, 2004.

EZZAHOUANI, A.; WILLIAMS, L.E. The influence of rootstock on leaf water potential, yield, and berry composition of Ruby Seedless grapevines. American Journal of Enology and Viticulture, v.46, p.559-563, 1995.

FAVERO, A.C.; AMORIM, D.A. de; MOTA, R.V. da; SOARES, Â.M.; REGINA, M. de A. Viabilidade de produção da videira 'Syrah', em ciclo de outono inverno, na região sul de Minas Gerais. Revista Brasileira de Fruticultura, v.30, p.685-690, 2008.

FERREIRA, F.D. SISVAR: um programa para análises e ensino de estatística. Revista Symposium, v.6, p.36-41, 2008.
GINESTAR, C.; EASTHAM, J.; GRAY, S.; ILAND, P. Use of sap-flow sensors to schedule vineyard irrigation. II. Effects of post-veraison water deficits on composition of Shiraz grapes. American Journal of Enology and Viticulture, v.49, p.421-428, 1998.

GIUSTI, M.M.; WROSLTAD, R.E. Characterization and measurement of anthocyanins by uv-visible spectroscopy. New York: John Willey \& Sons, 2000. (Current Protocols in Food Analytical Chemistry). Available at: <http://www.does.org/ masterli/facsample.htm>. Accessed on: 20 Mar. 2010.

GONZÁLEZ-NEVES, G.; CHARAMELO, D.; BALADO, J.; BARREIRO, L.; BOCHICCHIO, R.; GATTO, G.; GIL, G.; TESSORE, A.; CARBONNEAU, A.; MOUTOUNET, M. Phenolic potential of Tannat, Cabernet Sauvignon and Merlot grapes and their correspondence with wine composition. Analytica Chimica Acta, v.513, p.191-196, 2004.

JACKSON, D.I.; LOMBARD, P.B. Environmental and management practices affecting grape composition and wine quality - a review. American Journal of Enology and Viticulture, v.44, p.409-430, 1993.

KLIEWER, W.M.; DOKOOZLIAN, N.K. Leaf area/crop weight ratios of grapevines: influence on fruit composition and wine quality. American Journal of Enology and Viticulture, v.56, p.170-181, 2005.

KOUNDOURAS, S.; HATZIDIMITRIOU, E.; KARAMOLEGKOU, M.; DIMOPOULOU, E.; KALLITHRAKA, S.; TSIALTAS, J.T.; ZIOZIOU, E.; NIKOLAOU, N.; KOTSERIDIS, Y. Irrigation and rootstock effects on the phenolic concentration and aroma potential of Vitis vinifera L. cv. Cabernet Sauvignon grapes. Journal of Agricultural and Food Chemistry, v.57, p.7805-7813, 2009.

KOUNDOURAS, S.; TSIALTAS, I.T.; ZIOZIOU, E.; NIKOLAOU, N. Rootstock effects on the adaptive strategies of grapevine (Vitis vinifera L. cv. Cabernet-Sauvignon) under contrasting water status: leaf physiological and structural responses. Agriculture, Ecosystems and Environment, v.128, p.86-96, 2008.

MAIN, G.; MORRIS, J.; STRIEGLER, K. Rootstock effects on Chardonel productivity, fruit, and wine composition. American Journal of Enology and Viticulture, v.53, p.37-40, 2002.

MALAVOLTA, E.; VITTI, G.C.; OLIVEIRA, S.A. de. Metodologia para análise de elementos em material vegetal. In: MALAVOLTA, E.; VITTI, G.C.; OLIVEIRA, S.A. de. Avaliação do estado nutricional das plantas: princípios e aplicações. 2.ed. Piracicaba: POTAFOS, 1997. p.231-307.

MAZZA, G.; MINIATI, E. Anthocyanins in fruits, vegetables and grains. Boca Raton: CRC Press, 1993. 191p.

MCCORD, J.D.; TROUSDALE, E.; RYU, D.D.Y. An improved sample preparation procedure for the analysis of major organic components in grape must and wine by high performance liquid chromatography. American Journal of Enology and Viticulture, v.35, p.28-29, 1984.

ORTEGA-REGULES, A.; ROMERO-CASCALES, I.; ROS-GARCÍA, J.M.; BAUTISTA-ORTÍN, A.B.; LÓPEZ-ROCA, J.M.; FERNÁNDEZ-FERNÁNDEZ, J.I.; GÓMEZ-PLAZA, E. Anthocyanins and tannins in four grape varieties (Vitis vinifera L.). 
Evolution of their content and extractability. International Journal of Vine and Wine Sciences, v.42, p.147-156, 2008.

REGINA, M. de A.; AUDEGUIN, L. Avaliação ecofisiológica de clones de videira cv. Syrah. Ciência e Agrotecnologia, v.29, p.875-879, 2005.

REGINA, M. de A.; FRÁGUAS, J.C.; ALVARENGA, A.A.; SOUZA, C.R. de; AMORIM, D.A. de; MOTA, R.V. da; FÁVERO, A.C. Implantação e manejo do vinhedo para produção de vinhos de qualidade. Informe Agropecuário, v.27, p.16-31, 2006.

REGINA, M. de A.; PEREIRA, G.E.; CANÇADO, G.M.A.; RODRIGUES, D.J. Cálculo da área foliar em videira por método não destrutivo. Revista Brasileira de Fruticultura, v.22, p.310-313, 2000.

RIZZON, L.A.; MIELE, A. Avaliação da cv. Cabernet Sauvignon para elaboração de vinho tinto. Ciência e Tecnologia de Alimentos, v.22, p.192-198, 2002.
RIZZON, L.A.; MIELE, A. Avaliação da cv. Tannat para elaboração de vinho tinto. Ciência e Tecnologia de Alimentos, v.24, p.223-229, 2004.

SANTOS, H.G. dos; JACOMINE, P.K.T.; ANJOS, L.H.C. dos; OLIVEIRA, V.A. de; OLIVEIRA, J.B. de; COELHO, M.R.; LUMBRERAS, J.F.; CUNHA, T.J.F. (Ed.). Sistema brasileiro de classificação de solos. 2.ed. Rio de Janeiro: Embrapa Solos, 2006. $306 \mathrm{p}$.

SILVA, L.C. da; RUFATO, L.; KRETZSCHMAR, A.A.; MARCON FILHO, J.L. Raleio de cachos em vinhedos de altitude e qualidade do vinho da cultivar 'Syrah'. Pesquisa Agropecuária Brasileira, v.44, p.148-154, 2009.

TONIETTO, J.; VIANELLO, R.L.; REGINA, M.A. Caracterização macroclimática e potencial enológico de diferentes regiões com vocação vitícola em Minas Gerais. Informe Agropecuário, v.27, p.32-55, 2006.

Recebido em 21 de outubro de 2011 e aprovado em 23 de janeiro de 2012 